\title{
Development and evaluation of a Loop Mediated Isothermal Amplification (LAMP) technique for the detection of hookworm (Necator americanus) infection in fecal samples
}

Robert Muriuki Mugambi ${ }^{1,2}$, Eric L. Agola ${ }^{2 *}$, Ibrahim N. Mwangi ${ }^{2}$, Johnson Kinyua ${ }^{3}$, Esther Andia Shiraho ${ }^{2}$ and Gerald M. Mkoji ${ }^{2}$

\begin{abstract}
Background: Hookworm infection is a major concern in sub-Saharan Africa, particularly in children and pregnant women. Necator americanus and Ancylostoma duodenale are responsible for this condition. Hookworm disease is one of the Neglected tropical diseases (NTDs) that are targeted for elimination through global mass chemotherapy. To support this there is a need for reliable diagnostic tools. The conventional diagnostic test, Kato-Katz that is based on microscopic detection of parasite ova in faecal samples, is not effective due to its low sensitivity that is brought about mainly by non-random distribution of eggs in stool and day to day variation in egg output. It is tedious, cumbersome to perform and requires experience for correct diagnosis. LAMP-based tests are simple, relatively cheap, offer greater sensitivity, specificity than existing tests, have high throughput capability, and are ideal for use at the point of care.

Methods: We have developed a LAMP diagnostic test for detection of hookworm infection in faecal samples. LAMP relies on auto cycling strand displacement DNA synthesis performed at isothermal temperature by Bst polymerase and a set of 4 specific primers. The primers used in the LAMP assay were based on the second Internal Transcribed Spacer (ITS-2) region and designed using Primer Explorer version 4 Software. The ITS-2 region of the ribosomal gene (rDNA) was identified as a suitable target due to its low mutation rates and substantial differences between species. DNA was extracted directly from human faecal samples, followed by LAMP amplification at isothermal temperature of $63^{\circ} \mathrm{C}$ for $1 \mathrm{~h}$. Amplicons were visualized using gel electrophoresis and SYBR green dye. Both specificity and sensitivity of the assay were determined.

Results: The LAMP based technique developed was able to detect $N$. americanus DNA in faecal samples. The assay showed $100 \%$ specificity and no cross-reaction was observed with other helminth parasites (S. mansoni, A. lumbricoides or T. trichiura). The developed LAMP assay was $97 \%$ sensitive and DNA at concentrations as low as $0.4 \mathrm{fg}$ were amplified. Conclusion: The LAMP assay developed is an appropriate diagnostic method for the detection of $N$. americanus DNA in human stool samples because of its simplicity, low cost, sensitivity, and specificity. It holds great promise as a useful diagnostic tool for use in disease control where infection intensities have been significantly reduced.
\end{abstract}

Keywords: Loop mediated isothermal amplification, ITS-2, Necator americanus, Kato-Katz

\footnotetext{
* Correspondence: Elelo@kemri.org

${ }^{2}$ Center for Biotechnology Research and Development, Kenya Medical

Research Institute (KEMRI), P.O Box 54840, 00200 Nairobi, Kenya

Full list of author information is available at the end of the article
}

(c) 2015 Mugambi et al. Open Access This article is distributed under the terms of the Creative Commons Attribution 4.0 International License (http://creativecommons.org/licenses/by/4.0/), which permits unrestricted use, distribution, and reproduction in any medium, provided you give appropriate credit to the original author(s) and the source, provide a link to the Creative Commons license, and indicate if changes were made. The Creative Commons Public Domain Dedication waiver (http://creativecommons.org/publicdomain/zero/1.0/) applies to the data made available in this article, unless otherwise stated. 


\section{Background}

Hookworm infection in humans is caused by helminth nematode parasites Necator americanus and Ancylostoma duodenale and is transmitted through contact with contaminated soil. It is one of the most common chronic infections, with an estimated 740 million cases in the tropics and subtropics [1]. It is predominant among people living in poverty stricken areas [2]. The greatest number of hookworm cases occurs in Asia, followed by sub-Saharan Africa [1]. Globally, the most common hookworm species is $N$. americanus, while $A$. duodenale is more restricted geographically [3]. Hookworm infection is among the most important tropical diseases in humans. Disability-adjusted life years as a measure of the burden of disease reveals that this infection dominates African trypanosomiasis, dengue, Chagas' disease, schistosomiasis, and leprosy [4]. The most damaging effects of hookworm infections include impaired physical, intellectual and cognitive development of children, increased mortality in pregnant women and their infants and reduced work capacity of adolescents and adults [5].

Hookworm has a direct lifecycle. The infective stage is a third stage larva that penetrates the skin upon contact with contaminated soil. While in the soil the third stage larvae are in a state of developmental arrest; development continues after it penetrates the skin of the human host [5]. The major clinical manifestation of hookworm infection is due to the consequences of chronic intestinal blood loss resulting in iron-deficiency leading to anaemia. Hypoalbuminemia develops when blood loss exceeds the intake and reserves of host iron and protein. The degree of iron-deficiency anaemia induced by hookworms depends on the species; $A$. duodenale causes greater blood loss than infection with $N$. americanus [4].

The formulation of effective control measures for hookworm is reliant on accurate diagnosis. Currently, the diagnosis of hookworms and other soil transmitted helminths is by the use of microscopy for the identification of ova in faeces and third-stage larvae (L3) through the copromiscroscopic technique, more specifically Kato-Katz. Kato-Katz method of diagnosis is widely accepted because it is technically simple and requires very minimal costs to perform [6]. Despite this, it is limited in terms of specificity because it cannot differentiate between ova of the two human hookworm species, specifically $N$. americanus and $A$. duodenale. It is also not sensitive enough to detect low infection in an individual. Other than its lack of sensitivity and specificity, microscopy based diagnostic technique is laborious, time consuming and requires a skilled individual for correct diagnosis.

Molecular based techniques that are dependent on Polymerase chain reaction (PCR) are available and are more sensitive and specific than the parasitological techniques. However, these methods are very expensive because of their requirement for costly equipment. Loop mediated isothermal amplification technique (LAMP) is a technique developed by Notomi et al. that amplifies DNA with high specificity, efficiency and rapidity under isothermal conditions. This nucleic acid amplification technique uses only one enzyme (Bst DNA polymerase) and is able to amplify large amounts of DNA within 30-60 min by auto-strand displacement DNA synthesis [7]. The technique has several advantages including; 1) the reaction takes place at isothermal temperatures between $60{ }^{\circ} \mathrm{C}$ and $65^{\circ} \mathrm{C}$, and therefore simple incubators, such as a water bath or block heater, are sufficient for the DNA amplification, 2) LAMP is more stable than PCR and realtime PCR, 3) Non-involvement of template DNA preparation and ability to generate $10^{9}$ copies of DNA are added benefits that make it more effective ,4) It is also easy to visualize the amplicons because in LAMP assay, as amplification of DNA continues there is the formation of pyrophosphates that causes turbidity and thus makes visualization easy [8]. The product can also be visualized directly using simple detection methods such as adding DNA binding dye, SYBR green. Performance of LAMP mainly relies on designing Primers that should be very specific. LAMP requires a minimum of 4 primers namely; F3 (Forward outer), B3 (Backward outer), FIP (Forward inner) and BIP (Backward inner) primers. Two extra loop primers, LF (Loop forward) and LB (Loop backward) can also be assimilated which hastens the reaction, hence taking a shorter time [9].

Loop mediated isothermal amplification has emerged as a powerful tool for point-of-care diagnostics and has been used in diagnosis of protozoan parasitic diseases such as Plasmodium [10], S. haematobium [11], African Trypanosomiasis [12], S. japonicum [13] and Taenia species [14] with high efficacy.

We have developed a loop mediated isothermal technique assay that is quick, sensitive, and specific with minimal equipment requirement for the diagnosis of hookworm ( $N$. americanus) parasite in patient faecal samples.

\section{Methods}

\section{Study approval and ethical considerations}

This study was approved through the scientific and ethics committees of the Kenya Medical Research Institute (KEMRI). All the children found to be infected with hookworm or any of the soil-transmitted helminths received treatment with a single dose of $400 \mathrm{mg}$ albendazole under the supervision of a clinician. Faecal sample collection for STH infection diagnosis is a routine procedure for parasitological investigations and does not pose any risks or cause any harm to participating children. The samples of the study participants were coded and any data that was obtained from them was stored in a password protected 
personal computer accessible only to the Principal Investigator.

Faecal sample collection and parasitological examination Each participating child provided a single faecal sample in a capped plastic polypot, and these were transported back to the field laboratory in an ice box, within $2 \mathrm{~h}$ of collection, where duplicate Kato-Katz slides were prepared on glass microscope slides according to the standard procedure and examined under a compound microscope at x400 magnification, within $1 \mathrm{~h}$ of sample preparation. All hookworm positive samples were used for DNA extraction.

\section{DNA extraction}

Genomic DNA was extracted from freshly collected human stool samples using the QIAMP fast DNA stool mini kit from Qiagen, (catalog number 51604) following manufacturer instructions. Briefly, $0.2 \mathrm{~g}$ of stool sample was weighed and placed in $2 \mathrm{ml}$ tubes. $1 \mathrm{~mm}$ of inhibit EX buffer was then added to the tube containing the faecal sample and vortexed for $1 \mathrm{~min}$. The suspension was then heated for $5 \mathrm{mins}$ at $95{ }^{\circ} \mathrm{C}$. In a new $1.5 \mathrm{ml}$ tube, $15 \mu \mathrm{l}$ proteinase $\mathrm{K}$ was added. The supernatant was then added into the tube containing $15 \mu \mathrm{l}$ of proteinase $\mathrm{K}$, and centrifuged. Two hundred microliters of buffer AL was then added and the mixture vortexed for $15 \mathrm{~s}$. This was later incubated in a water bath for $10 \mathrm{mins}$ at $70{ }^{\circ} \mathrm{C}$ followed by addition of $200 \mu \mathrm{l}$ of absolute ethanol and thorough mixing. From this mixture $600 \mu \mathrm{l}$ of the lysate was pipetted into a spin column. This was centrifuged for $1 \mathrm{~min}$ at $14000 \mathrm{rpm}$ and the filtrate discarded. At this point, $500 \mu \mathrm{l}$ of buffer 1 (AW1) was added and centrifuged for $1 \mathrm{~min}$ and the filtrate discarded. $500 \mu \mathrm{l}$ buffer 2(AW2) was then added and centrifuged for 3 mins at $14000 \mathrm{rpm}$. The filtrate was then discarded and $200 \mu \mathrm{l}$ elution buffer (ATE) added and incubated at room temperature for $1 \mathrm{~min}$ and centrifuged for $1 \mathrm{~min}$ at $14000 \mathrm{rpm}$ to elute the DNA. The DNA was stored at $-20{ }^{\circ} \mathrm{C}$ until used.

\section{Primer design}

LAMP Primers were designed using Primer Explorer version 4.0 software (http://primerexplorer.jp/elamp4.0.0). The internal transcribed spacer (ITS-2) gene was chosen for amplification because of the lower mutation rates and substantial differences between species. A total of 18 sequences of internal transcribed spacer 2 were selected from the National Centre for Biotechnology information (NCBI). The accession numbers of these sequences are KC896820.1-KC 896825.1, Y11734, AF217891, HQ452515, HQ455217, HQ452537-HQ452543 and AJ001599. These sequences were subjected to multiple sequence alignment (MSA) using a program called CLC main workbench version 7.0 and a consensus sequence was developed, from which five sets of primers were developed. The best set of primers was selected after being tested for their suitability and reliability. The four sets of primers (two outer and two inner primers) were designed to recognize six separate regions within the ITS-2 gene. Primers were validated using BLAST software (http://www.ncbi.nlm.nih.gov/BLAST) to ensure that they were specific to $N$. americanus. Primer sequences are listed in Table 1.

\section{LAMP reaction}

The LAMP reaction was performed as described by [7]. Briefly, in a total reaction volume of $18 \mu \mathrm{l}$, 40pmoles of each inner primer (FIP and BIP) and 5pmoles of each outer primer (F3 and B3), $12.5 \mu$ l of $2 \mathrm{X}$ LAMP buffer, $2.5 \mu \mathrm{l}$ of Bst buffer, 1 unit of Bst polymerase and $2.0 \mu \mathrm{l}$ of DNA template were added. Amplification was carried out at $63{ }^{\circ} \mathrm{C}$ for one hour, followed by reaction termination at $80{ }^{\circ} \mathrm{C}$ for $10 \mathrm{~min}$.

\section{LAMP product detection}

As part of the assay development, the LAMP products were initially detected by several methods, including electrophoresis on a $2.0 \%$ agarose gel stained with $2 \mu \mathrm{l}$ ethidium bromide and photographed using a gel documentation system. SYBR green dye was also used for the detection of amplicons using the naked eye. In the presence of positive DNA amplification SYBR green changes colour from orange to green. One microliter of SYBR green I dye was added to each tube containing LAMP products.

The specificity and sensitivity of the LAMP assay

To verify the specificity of the LAMP assay for the detection of $N$. americanus DNA, the LAMP primers specific for hookworm were tested using DNA from other related helminths i.e. S. mansoni, A. lumbricoides and T. trichiura. In order to determine the sensitivity of the LAMP assay, $10 \mathrm{ng} / \mathrm{ul}$ of $N$. americanus DNA was successively diluted 10 times by the addition of $1 \mathrm{ul}$ of a $1 / 10$ dilution of the previous concentration.

\section{Results}

Primer and temperature selection for the LAMP reaction In this study, we designed 5 sets of primers for LAMP amplification. Among these primers, those that had the

Table 1 Nucleotide sequences of the LAMP primers targeting the ITS-2 gene of N. americanus

\begin{tabular}{ll}
\hline Primer & Sequence \\
\hline FIP & CAC TTA AAC GGG AAT TGC ACC GAA CGG TAC TTG CTC TG \\
BIP & GCA ACA TGTGCA CGC TGT TAA CAG TAT GCA CCG CTA TC \\
F3 & AGT ATT GTT GAA CAC TGT TTTG T \\
B3 & AAC AAC GAT ATG TTC ATG TCA T \\
\hline
\end{tabular}

Kato-Katz (gold standard) 
highest amplification rates and the shortest amplification time were selected for analysis (Table 1). To optimize the LAMP reaction temperature, we conducted the procedures under different temperature conditions. LAMP assays were performed under isothermal conditions between $59{ }^{\circ} \mathrm{C}$ and $66{ }^{\circ} \mathrm{C}$. Based on the larger amounts of DNA amplicons and the optimal temperature for Bst DNA polymerase activity, we choose $63{ }^{\circ} \mathrm{C}$ as the final reaction temperature for our LAMP assay. Amplification was completed within $1 \mathrm{~h}$ isothermally at $63{ }^{\circ} \mathrm{C}$ in a water bath. The products of the LAMP reaction were detected by SBYR green nucleic acid stain where the positive samples changed colour from orange to green while the negative control remained orange (Fig. 1). The products were also viewed on a $2 \%$ agarose gel and showed ladder-like patterns (Fig. 2).

\section{Specificity of the LAMP reaction}

To determine specificity of the primers, two hookworm isolates were subjected to the LAMP assay alongside samples positive by Kato-Katz technique for other helminths i.e. S. mansoni, A. lumbricoides, and T. trichiura. These were amplified using primers specific for hookworm (N. americanus). As shown (Fig. 3), LAMP products were amplified only from DNA samples of $N$. americanus and no false positive amplification was observed, indicating the high specificity of the established LAMP assays.

\section{Sensitivity of the LAMP reaction}

Tenfold serial dilutions of total genomic DNA from $N$. americanus were used to test the sensitivity of the LAMP assay. The results indicated that the detection limit for the LAMP reaction was $0.4 \mathrm{fg}$ of DNA (Fig. 4).

\section{Comparison of Loop mediated isothermal amplification (LAMP) against Kato-Katz}

A total of 106 stool samples from the field were analysed and the results tabulated as shown (Table 2). Out of the 106 samples, 86 samples were found to be positive by Kato-Katz technique, while 83 of the positive samples were found to be positive by LAMP. 20 samples were found to be negative for hookworms by both Kato-Katz and LAMP techniques.

In terms of sensitivity LAMP showed $97 \%$ while in terms of specificity LAMP showed $100 \%$. We also found the agreement between the two techniques by calculating the Kappa coefficient as described by Viera et al. [15], which is calculated as follows

$$
\begin{aligned}
\text { Kappa coefficient }= & (\% \text { Observed Agreement. }) \\
& -(\% \text { agreement Expected by chance }) \\
= & \mathbf{0 . 9}
\end{aligned}
$$

$100 \%$ - (\% agreement Expected by chance)

The Kappa coefficient was 0.9, which shows an excellent agreement between the two techniques, thus proving LAMP to a reliable diagnostic tool for hookworms.

\section{Discussion}

In this study, we reported on LAMP, a novel sensitive and rapid detection method for diagnosis of hookworm caused by Necator americanus. An accurate, specific and sensitive diagnosis system for hookworm is critical for accurate detection of hookworm following mass drug administration when infection intensities are lowered following chemotherapy. The current WHO recommended strategies in the control of schistosomiasis and STHs is by mass drug administration (MDA). One notable result of MDA is the lowering of infection intensity both in individuals and populations under treatment.

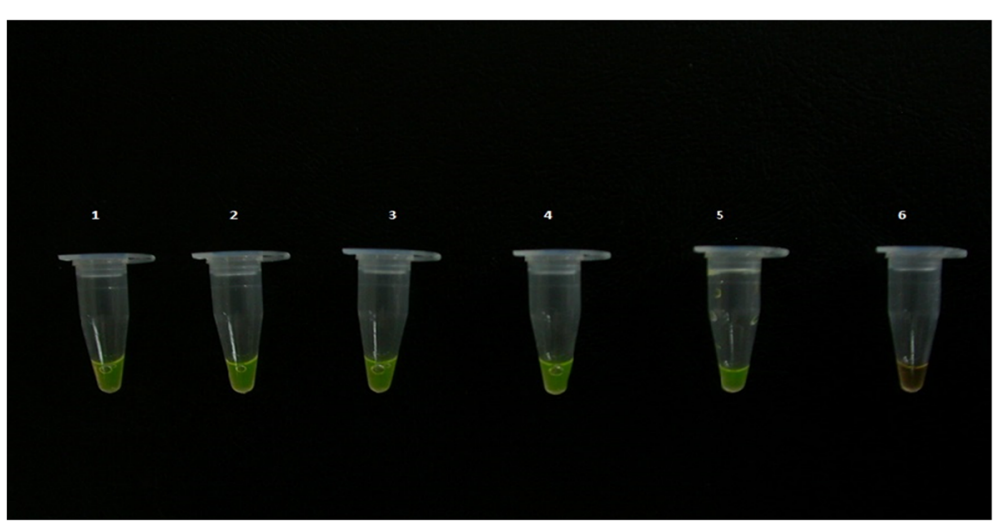

Fig. 1 A photo showing the colour change using SYBR green nucleic acid stain as a method of visualizing LAMP amplicons. Samples 1 to 5 were positive for hookworms hence the observed colour change from orange to green, while sample 6 was the negative control thus there was no colour change 


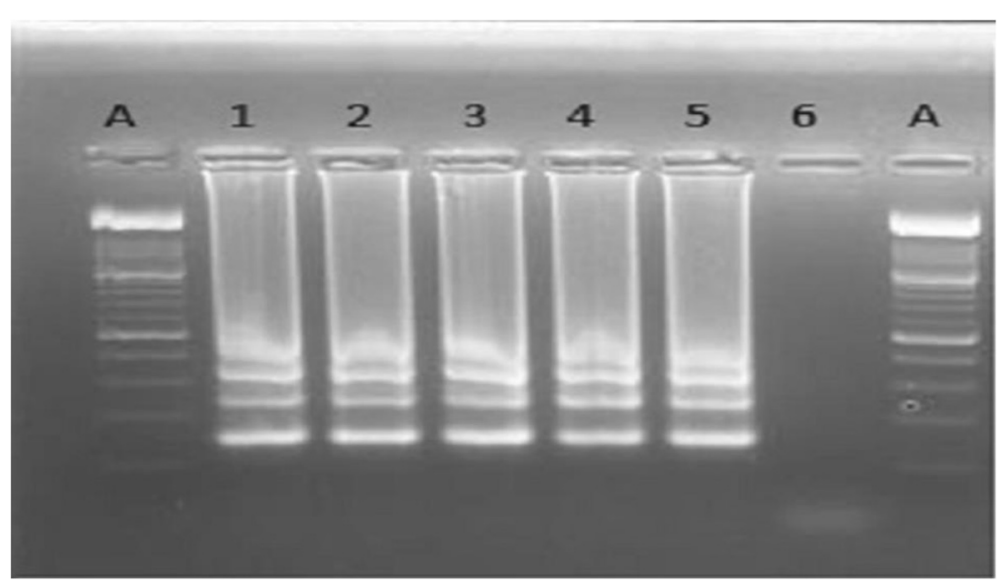

Fig. 2 Agarose gel showing the fragments amplified from human faecal samples containing N. americanus DNA (lane 1-5). Lane 6 is the negative control (PCR water). A represents a 100-bp ladder

This requires diagnostic methods that are sensitive enough to detect infections at very low intensities. Parasitological diagnostic methods such as Kato-Katz have commonly been used to diagnose STHs but these methods have the disadvantage of low sensitivity mainly due to non-random egg distribution in faecal samples, hence making the monitoring of the success of control programmes a challenge. Molecular based techniques that are dependent on Polymerase chain reaction (PCR) are available and are more sensitive and specific than the parasitological techniques. However, these methods are very expensive because of their requirement for costly equipment. Loop mediated isothermal amplification technique (LAMP) is a technique developed by Notomi et al., [7] that amplifies DNA with high specificity, efficiency and rapidity under isothermal conditions.
In this study we have developed a LAMP assay that can detect hookworm DNA in faecal human samples. The developed LAMP technique was based on the internal transcribed spacer 2 (ITS-2) of the nuclear ribosomal DNA. This region was chosen as a potential marker because of its low mutation rates within species and substantial differences between species [16], hence making it an excellent candidate for designing LAMP primers for detection of hookworms. The primers designed were highly specific and only amplified DNA of $N$. americanus and had no cross-reaction with nucleic acid from closely related species.

LAMP has the characteristics that make it ideal for field work and has already been used in rural laboratories in developing areas for the diagnosis of tuberculosis [17]. One advantage of a LAMP test over the conventional

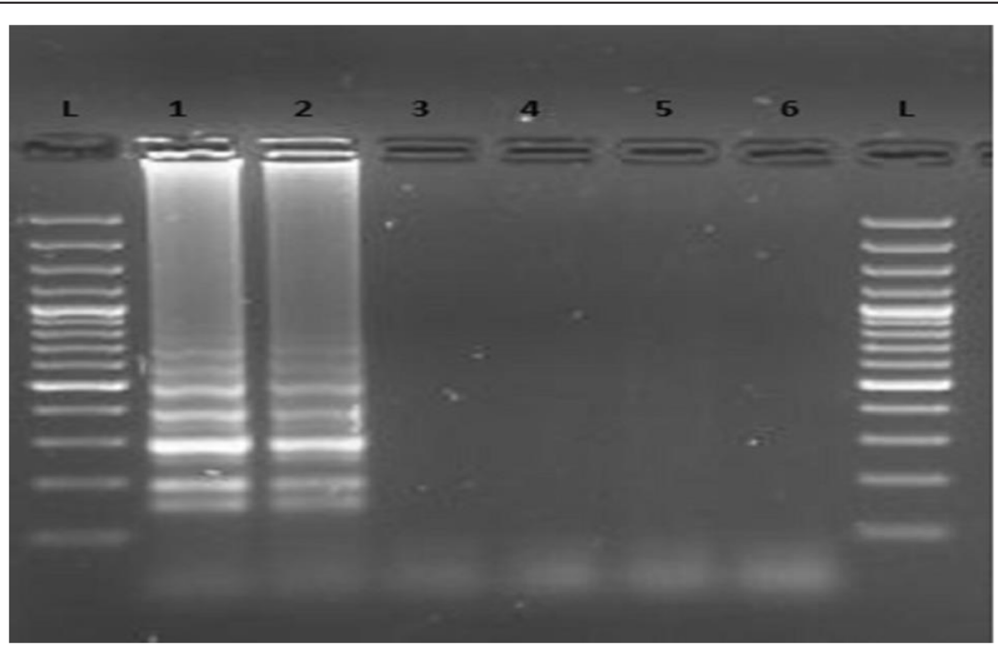

Fig. 3 Agarose gel showing the specificity of the test, N. americanus DNA (lane 1 \& 2), S. mansoni DNA (Lane 3), A. lumbricoides DNA (Lane 4), T. trichiura DNA (Lane 5) and Lane 6 is the negative control (PCR water). L represents a 100-bp ladder 


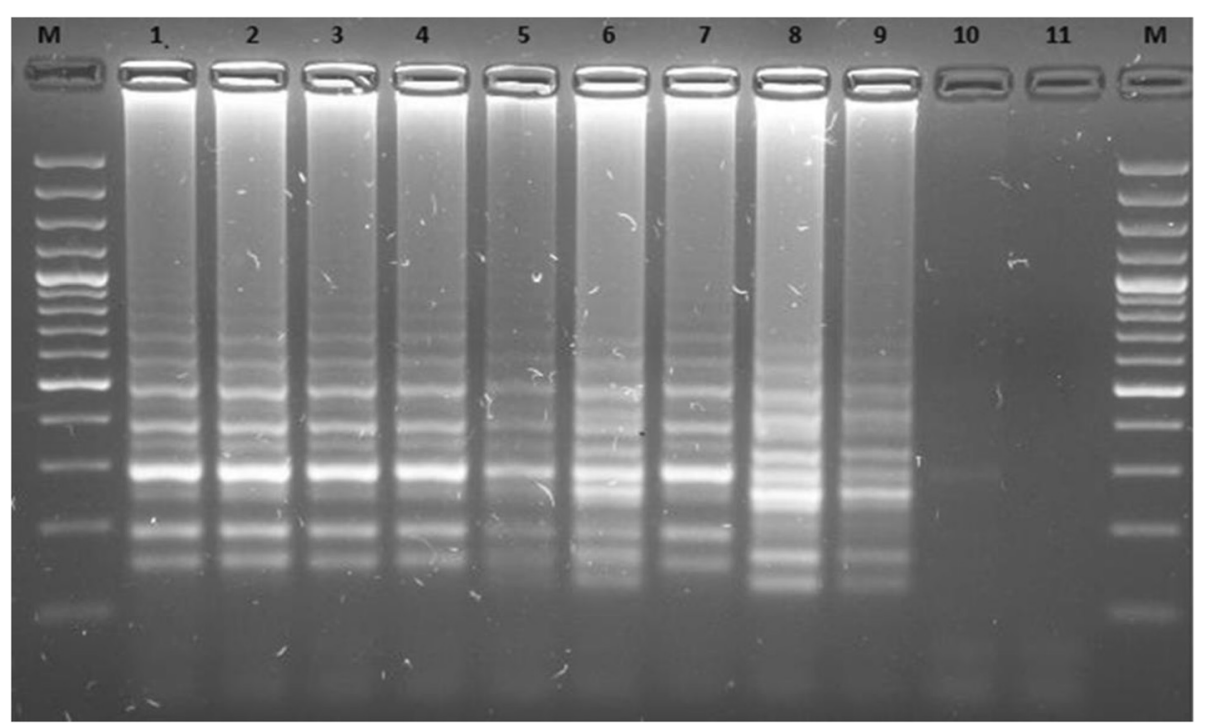

Fig. 4 Agarose gel electrophoresis of loop-mediated isothermal amplification (LAMP) products from different concentrations of Necator americanus genomic DNA. Ten-fold serial dilutions starting with $10 \mathrm{ng}$ of genomic DNA (lane 1) down to $0.4 \mathrm{fg}$ (lane 10) were tested. Lane 11, negative control (no DNA); lane M molecular marker

PCR is that sophisticated equipment required for PCR, is not required for the LAMP assay, a simple detection system based on staining the amplification products with SYBR green dye, which can be visualized with the naked eye, demonstrating the feasibility of using this assay under field conditions. In the present study, we were able to detect the amplified DNA not only using agarose gel but also with the naked eye following staining with SYBR green dye. Because the assay reaction is isothermal, a simple water bath can be used for this test. Furthermore, the fact that the developed assay could detect parasite DNA concentrations as low as $0.4 \mathrm{fg}$ suggests that even low intensity of $N$. americanus infections can readily be detected using the developed LAMP assay. The Kato-Katz technique diagnosed three samples as positive but the same samples were negative by LAMP technique. We attributed this difference to human error since our control samples were always in agreement with the LAMP results. Kato-Katz technique relies on expert microscopy that is subjective and due to a number of factors such as technical experience, eye sight, preparation of the stool smears among other factors, therefore reading may differ from

Table 2 Contingency table comparing LAMP technique with Kato-Katz (gold standard)

\begin{tabular}{llll}
\hline & + & - & Totals \\
\hline+ & 83 & 0 & 83 \\
- & 3 & 20 & 23 \\
Totals & 86 & 20 & 106 \\
\hline
\end{tabular}

LAMP (Screen test) one reader to the other. Among the other tests available for the diagnosis of hookworms is the Mini-FLOTAC device which is the most promising. However, a recent meta-analysis and field studies comparing it's sensitivity with that of Kato-Katz have shown no significant difference [18-20].

We recommend further development of the technique to make it adaptable for application in the field laboratory set up. Specifically, the DNA extraction kit should be replaced with a simpler and inexpensive DNA extraction method. Also the LAMP reagents would need to be premixed and ready to use to avoid too many pipetting steps that may result in sample contamination; moreover reagents that can be stored at room temperature need to be formulated as this could facilitate their viability under field-laboratory conditions.

\section{Conclusion}

In summary, in the current study, we showed that the LAMP technique based on the ITS-2 region enables specific detection of $N$. americanus but excludes related helminth species. It is a novel technique that can potentially be used for rapid diagnosis of $N$. americanus infections, not only in laboratories but also in a field setting. In conclusion, the LAMP method described in this study represents a new, sensitive, specific, and rapid protocol for the detection of $N$. americanus and has the potential for point of care application. The findings of this present study demonstrate that this technique can be applied to epidemiological studies to detect hookworm prevalence and infection intensities in infected individuals. 


\section{Competing interests}

The authors declare that no competing interest exists.

\section{Authors' contribution}

$\mathrm{RM}$; He was involved in every aspect of the study. He was also responsible in conception and design of the study, data collection and interpretation. He also made significant contribution in drafting the manuscript. EL; He was involved in conception and designing the study. He made significant contribution to acquisition of data and also its analysis. He was involved in drafting the manuscript and also critically revising it. JK; He played a critical role in designing the study. He also made significant contribution in critically revising the manuscript before submission GM; He was involved in conceptualizing and designing the study. He also made contribution in revising the manuscript. IM and ES; they were involved in acquisition and interpretation of data. All authors read and approved the final version of the manuscript.

\section{List of financial support}

This study was supported through funding provided by Grand Challenges Canada, Grant No. 0261-01 to ALE and GMM, and is published with the approval of Director, KEMRI.

\section{Author details}

${ }^{1}$ Institute of Tropical Medicine and Infectious Diseases, Jomo Kenyatta University of Agriculture and Technology (JKUAT), P.O. Box 54840, 00200 Nairobi, Kenya. ${ }^{2}$ Center for Biotechnology Research and Development, Kenya Medical Research Institute (KEMRI), P.O Box 54840, 00200 Nairobi, Kenya. ${ }^{3}$ Department of Biochemistry, Jomo Kenyatta University of Agriculture and technology (JKUAT), P.O. Box 62000, 00200 Nairobi, Kenya.

Received: 9 July 2015 Accepted: 30 October 2015

Published online: 06 November 2015

\section{References}

1. Hotez PJ. One world health: neglected tropical diseases in a flat world. PLoS Negl Trop Dis. 2009;3(4).

2. Hotez P. Hookworm and poverty. Ann N Y Acad Sci. 2008;1136:38-44.

3. De Silva NR, Brooker S, Hotez PJ, Montresor A, Engels D, Savioli L. Soil-transmitted helminth infections: updating the global picture. Trends Parasitol. 2003;19(12):547-51.

4. Hotez PJ, Zhan B, Bethony JM, Loukas A, Williamson A, Goud GN, et al. Progress in the development of a recombinant vaccine for human hookworm disease: the human hookworm vaccine initiative. Int J Parasitol. 2003;33(11):1245-58.

5. Brooker S, Bethony J, Hotez PJ. Human hookworm infection in the $21 \mathrm{st}$ century. Adv Parasitol. 2004;58:197-288.

6. Ngui R, Ching LS, Kai TT, Roslan MA, Lim YAL. Molecular identification of human hookworm infections in economically disadvantaged communities in Peninsular Malaysia. Am J Trop Med Hyg. 2012;86(5):837-42.

7. Notomi T, Okayama H, Masubuchi H, Yonekawa T, Watanabe K, Amino N et al. Loop-mediated isothermal amplification of DNA. Nucleic Acids Res. 2000;28:E63.

8. Dhama K, Karthik K, Chakraborty S, Tiwari R, Kapoor S, Kumar A, et al. Loop-mediated isothermal amplification of DNA (LAMP): a new diagnostic tool lights the world of diagnosis of animal and human pathogens: a review. Pakistan J Biol Sci. 2014;17(2):151-66.

9. Nagamine $K$, Hase T, Notomi T. Accelerated reaction by loop-mediated isothermal amplification using loop primers. Mol Cell Probes. 2002;16(3):223-9.

10. Han ET, Watanabe R, Sattabongkot J, Khuntirat B, Sirichaisinthop J, Iriko H, et al. Detection of four Plasmodium species by genus- and species-specific loop-mediated isothermal amplification for clinical diagnosis. J Clin Microbiol. 2007:45(8):2521-8.

11. Abbasi I, King CH, Muchiri EM, Hamburger J. Detection of Schistosoma mansoni and Schistosoma haematobium DNA by loop-mediated isothermal amplification: identification of infected snails from early prepatency. Am J Trop Med Hyg. 2010;83(2):427-32.

12. Njiru ZK, Mikosza ASJ, Matovu E, Enyaru JCK, Ouma JO, Kibona SN, et al. African trypanosomiasis: sensitive and rapid detection of the sub-genus trypanozoon by loop-mediated isothermal amplification (LAMP) of parasite DNA. Int J Parasitol. 2008;38(5):589-99.
13. Xu J, Rong R, Zhang $H Q$, Shi CJ, Zhu XQ, Xia CM. Sensitive and rapid detection of Schistosoma japonicum DNA by loop-mediated isothermal amplification (LAMP). Int J Parasitol. 2010;40(3):327-31.

14. Nkouawa A, Sako Y, Li T, Chen X, Wandra T, Swastika IK, et al. Evaluation of a loop-mediated isothermal amplification method using fecal specimens for differential detection of Taenia species from humans. J Clin Microbiol. 2010;48(9):3350-2.

15. Viera AJ, Garrett JM. Understanding interobserver agreement: the kappa statistic. Fam Med. 2005;37(5):360-3.

16. Ngui R, Lim YAL, Chua KH. Rapid detection and identification of human hookworm infections through high resolution melting (HRM) analysis. PLoS One. 2012;7:7.

17. Boehme CC, Nabeta P, Henostroza G, Raqib R, Rahim Z, Gerhardt M, et al. Operational feasibility of using loop-mediated isothermal amplification for diagnosis of pulmonary tuberculosis in microscopy centers of developing countries. J Clin Microbiol. 2007:45(6):1936-40.

18. Assefa LM, Crellen T, Kepha S, Kihara JH, Njenga SM, Pullan RL, et al. Diagnostic accuracy and cost-effectiveness of alternative methods for detection of soil-transmitted helminths in a post-treatment setting in western Kenya. PLoS Negl Trop Dis. 2014;8(5):e2843.

19. Barda B, Zepherine H, Rinaldi L, Cringoli G, Burioni R, Clementi M, et al. Mini-FLOTAC and Kato-Katz: helminth eggs watching on the shore of Lake Victoria. Parasit Vectors. 2013;6(1):220.

20. Nikolay B, Brooker SJ, Pullan RL. Sensitivity of diagnostic tests for human soil-transmitted helminth infections: a meta-analysis in the absence of a true gold standard. Int J Parasitol. 2014;44(11):765-74.

\section{Submit your next manuscript to BioMed Central and take full advantage of:}

- Convenient online submission

- Thorough peer review

- No space constraints or color figure charges

- Immediate publication on acceptance

- Inclusion in PubMed, CAS, Scopus and Google Scholar

- Research which is freely available for redistribution 